\title{
DEPRESSION, ANXIETY, PERCEIVED AND POST-TRAUMATIC STRESS, AND THE USE OF ALCOHOL DURING THE COVID-19 PANDEMIC IN ESTONIA FROM SPRING TO AUTUMN IN 2020: A LONGITUDINAL STUDY
}

\author{
Karel Kulbin, Kristjan Kask, and Kristiina Uriko
}

Tallinn University

\begin{abstract}
The situation regarding the COVID-19 pandemic has changed quickly due to changing case numbers and changing governmental restrictions indicating that mental health consequences may also have changed rapidly and differed by countries. The aim of our study was to describe and map changes in the scores of depression, anxiety, perceived and post-traumatic stress, and the use of alcohol in Estonia during the COVID-19 pandemic. The three waves of data collection were conducted $(\mathrm{N}=202)$ in spring $(\mathrm{T} 1)$, summer (T2) and fall (T3) in 2020. The survey included information about the study, a set of demographic, health and wellness questions, and a set of self-reported scales about mental health and wellbeing. At T3 there were a larger proportion of respondents whose Anxiety and Fatigue scores were above the cut-off point compared to T2. At T2 there were a larger proportion of respondents whose AUDIT-C score was above the cut-off point compared to T3. At T1 there was a larger proportion of respondents whose Agoraphobia and panic score was above the cut-off point compared to T3. This is the first Estonian longitudinal study mapping changes in mental health during the COVID-19 pandemic.
\end{abstract}

Keywords: COVID-19, perceived stress, alcohol abuse, depression, anxiety, mental health

DOI: https//doi.org/10.3176/tr.2021.3.01

Received 25 May 2021, accepted 5 August 2021, printed and available online 10 September 2021

(C) 2021 Authors. This is an Open Access article distributed under the terms and conditions of the Creative Commons Attribution-NonCommercial 4.0 International License (http://creativecommons.org/licenses/by-nc/4.0/). 


\section{Introduction}

Many countries face challenges in response to the COVID-19 pandemic. The countries have applied different measures in fighting the new coronavirus and its consequences. Public health response decisions were made under scientific uncertainty around the infectiousness and natural history of COVID-19 to protect the population and avoid overwhelming the health system. Since the beginning of the pandemic in 2020, mental health has been addressed as one of the research priorities (Holmes et al. 2020). As yet, we lack information on the response in this pandemic. Alot of the interest has focused on short-term concerns, but the mental health impact of the pandemic is likely to last much longer than the physical health impact (Kousoulis et al. 2020). Thus, it is important to document, if and what changes there have been in people's mental health since the beginning of and during the ongoing pandemic. The situation regarding the COVID-19 pandemic in different countries has changed quickly due to changing case numbers, changing governmental restrictions (Hale et al. 2020), habituation or change in media coverage. This indicates that mental health consequences may have changed also rapidly and differed by countries.

The reliable quantifications of the impact that the pandemic has had on mental health comes from a number of longitudinal cohort studies which have collected data from the same participants before and during the pandemic. Daly et al. (2020) found in a large nationally representative sample, that non-specific mental health problems increased substantially as the pandemic emerged in the UK and sustained throughout April to June 2020. Similarly, Pierce et al. (2020) found that by April 2020 there was an overall increase in mental distress in people aged 16 years and older in the UK compared with in the previous year. Mental distress did not affect all demographic groups equally - being young, a woman, and living with (especially preschool age) children had a particularly strong influence on the increase of mental distress during the first phase of the pandemic.

Another study from the UK has shown on a longitudinal population cohort that anxiety symptoms increased and wellbeing decreased during the lockdown of COVID-19 (April/May 2020) compared to pre-pandemic levels, particularly in young people (Kwong et al. 2020). Yet, they did not find clear evidence that prevalence of depressive symptoms had increased during the initial phase of COVID-19 from pre-pandemic levels. Gonzalez-Sanguino et al (2021) conducted a longitudinal study starting after one week into the state of emergency in Spanish population. They found that depressive symptoms increased throughout the confinement and did not drop to previous levels with de-escalation on the restrictive measures by the end of May 2020. On the other hand, the symptomatology of post-traumatic stress disorder (PTSD) showed a reduction with the lifting of the lockdown. Regarding the symptoms of anxiety, they found no significant changes between the three evaluations, from March to the end of May 2020. Their results also indicated that mental health of young people and females was more affected during the first stage of pandemic.

Similar studies have monitored their longitudinal samples further into the summer 
and/or early fall 2020, which has added mixed evidence regarding the longer lasting effects of pandemic on mental health. A longitudinal study of US adults also reported an initial increase in distress when pandemic emerged, but distress reduced close to pre-pandemic levels by June 2020 (Daly and Robinson 2021). Another study in England analyzed the trajectories of disorder-specific symptoms of anxiety and depression over the 20 weeks after lockdown (Fancourt et al. 2021). Their results showed that the highest levels of depressive and anxiety symptoms occurred in the beginning of lockdown but declined fairly rapidly. Both studies suggested that possibly individuals adapted to circumstances and resilience in mental health may have occurred in response to the pandemic (Daly and Robinson 2021, Fancourt et al. 2021). Bendau et al. (2021) conducted a research involving four data collection waves from March to June 2020 in Germany. They indicated that specific COVID19-related anxiety and the average daily amount of preoccupation with the pandemic decreased continuously over the four waves. Unspecific anxiety and especially depressive symptoms showed a slighter decrease on average. Controversially, a study in Austrian representative sample (Pieh et al. 2021) does not support these trends and found that detrimental mental health consequences persisted several months after the outbreak of pandemic. The prevalence of clinically relevant symptoms of depression, anxiety, or insomnia had not decreased six months after the outbreak of pandemic (by September 2020) in Austrian sample. Albeit they documented a slight trend toward improvement in perceived stress and well-being during the same period of time.

Perhaps the strongest evidence on the topic is lately presented in a systematic review and meta-analysis by Robinson et al. (2021). Their meta-analysis across 65 longitudinal cohort studies showed a statistically small overall increase in mental health symptoms, which was most pronounced during the first two months of the pandemic (March-April 2020), before generally decreasing close to pre-pandemic levels by mid-2020. More specifically, the increases in depressive symptoms tended to be larger and remained higher past the early phases of the pandemic, compared to anxiety symptoms, non-specific general mental health functioning and well-being. Detrimental consequences in mental health were observed across most population subgroups and change in mental health symptoms did not differ based on age. Contrary to predictions, their meta-analysis showed no evidence of a worsening of symptoms among samples with a pre-existing mental health condition. Somewhat interestingly, they observed similar patterns of increase in mental health symptoms both in North American and European samples (very few samples were included in their study from elsewhere). They found no evidence that country-level data (number of COVID cases/deaths; stringency of government measures) explained heterogeneity between samples. It seems plausible for the first stage of the pandemic, while governments in North America and Europe applied relatively similar restrictive measures with similar timings, regardless of largely differing COVID-19 case numbers, hospitalizations and deaths (Hale et al. 2020). Robinson et al. (2021) concluded that the first wave of pandemic (Spring 2020) may not have caused a long-lasting mental health crisis and the initial consequences were probably followed by a period of psychological adaptation and resilience. However, they call for continued mental health monitoring, 
given that there have been second waves of rapid increase of COVID-19 infection in many countries during late 2020 or early 2021.

A review of psychological stressful experiences and alcohol intake reports that stress is associated with increased risk for alcohol use, alcohol-related problems, and alcohol use disorders (Keyes et al. 2011). Recent COVID-19 studies reported some increase in alcohol use due to higher levels of stress, anxiety, and depression symptoms (Stanton et al. 2020, Oksanen et al. 2020, Newby et al. 2020, Ahmed et al. 2020). The Finnish longitudinal study outlines that previous hazardous drinkers tended to increase their drinking further (Oksanen et al. 2020). Being at home/ quarantined, lack of routine and symptoms of mental disorders were associated with increase in alcohol use. Excessive alcohol use can in turn lead to or worsen existing mental health problems, such as anxiety or depression. However, the cross-sectional online European Alcohol and COVID-19 survey, conducted between April 24 and July 22 of 2020, reported a lowered level of consumption (except in Ireland and UK) (Kilian et al. 2021). Declines in consumption were less marked among those experiencing distress. The findings suggest that both reduced physical and financial availability of alcohol may have affected alcohol consumption.

As there are two opposing views on whether alcohol consumption will increase during the COVID-19, it is meaningful to monitor how this public health crisis affects alcohol consumption. These results will provide more evidence of changes in alcohol use and associated consequences during the COVID-19 pandemic.

\subsection{Estonian context}

The World Health Organization (WHO) declared the COVID-19 pandemic on March 11, 2020 (WHO, 2020). In Estonia, the first COVID-19 case was diagnosed on February 26, 2020. The State of Emergency (SoE) was declared on March 12 which resulted in banning public gatherings, decreasing the availability of medical services (i.e. stopping regular treatments in hospital except urgent care) as well as closing all education facilities and transferring students to distance learning, closing all leisure facilities (excluding parks and recreational trails) along with restrictions in travelling to/from the islands and to/from abroad. Visits to hospitals and nursing homes were prohibited. Since March 25, the Government decided that there should be a maximum of two people being together in public places (or more, if they are of the same family) keeping a 2-metre distance with others both out- and indoors. Large shopping centers were closed from March 27. Open-air museums were opened again on May 2 and the SoE ended on May 18. The second wave of the virus arrived in November 2020 (Figure 1). The Health Board suggested people wear a mask in crowded places and to download the HOIA app to their smartphones to warn people early of a potential risk of having been infected. In addition, the Health Board organized a four-week social responsibility-oriented campaign 'Let's keep Estonia open!'. In December 2020, new national restrictions were approved. Until the end of 2020, all educational institutions were closed (kindergartens and childcare institutions remained open), only outdoor sports were allowed in groups of up to ten, holding public events were prohibited and all entertainment facilities closed for three weeks. 


\subsection{Current study}

The initial changes in mental health from the beginning of the pandemic until mid-2020 are relatively well-documented. However, there is so far a lack of evidence on the later fluctuations in mental health, as the second wave of COVID-19 and introduction of strict restrictions were widely re-experienced at differing time points onwards autumn 2020 in many countries. Thus, the aim of our study was to describe and map changes in the scores of depression, anxiety, perceived and post-traumatic stress, and the use of alcohol in Estonia during the COVID-19 pandemics in three data collection waves from spring to autumn in 2020 as currently mental health has only been studied among Estonian elite athletes in one timepoint from May to June 2020 (Parm et al. 2021). Also, as several studies have suggested that the mental health of younger adults has been more affected by the pandemic (e.g. Pierce et al. 2020, Kwong et al. 2020, Gonzalez-Sanguino et al. 2021), we were interested in how the age was associated with the mental health constructs at different time-points during the pandemic.

\section{Method}

\subsection{Sample}

All three waves of our survey were responded by 202 participants (183 females), mean age $45.56(S D=13.60$, range 18 to 81$)$. Of marital status, $36(18 \%)$ were single, 82 $(41 \%)$ were married, $12(6 \%)$ were divorced, $55(27.5 \%)$ were in an open relationship, $7(3.5 \%)$ in civil partnership and $12(6 \%)$ widowed (two participants did not answer this question). In terms of education, 1 had graduated basic school, 43 (21\%) vocational school or high school, 156 (78\%) higher education (two participants did not answer this question). The native language for 199 (98.5\%) participants was Estonian, for two participants Russian, and for one participant other language (not specified).

\subsection{Procedure}

The web-based survey created in SurveyMonkey environment was distributed in Estonian to potential participants through Tallinn University's homepage and social media channels, and also by banner in one of the largest news portals in Estonia in three data collection waves.

The three waves of data collection in a convenience sample were conducted (see Figure 1). The first period of data collection (T1) was open from April 20th until May 11th, involving 530 participants who consented to participate also in the next data collection waves. These participants were re-contacted in June, and $N=288$ (response rate of 54.3\%) answered the second survey (T2) from June 10th until June 26th. The third data collection (T3) was conducted from November 9th until December 6th, when $N=202$ (response rate of $70.1 \%$ of those who answered both at $\mathrm{T} 1$ and $\mathrm{T} 2$ ) participated for the third time in the survey. 


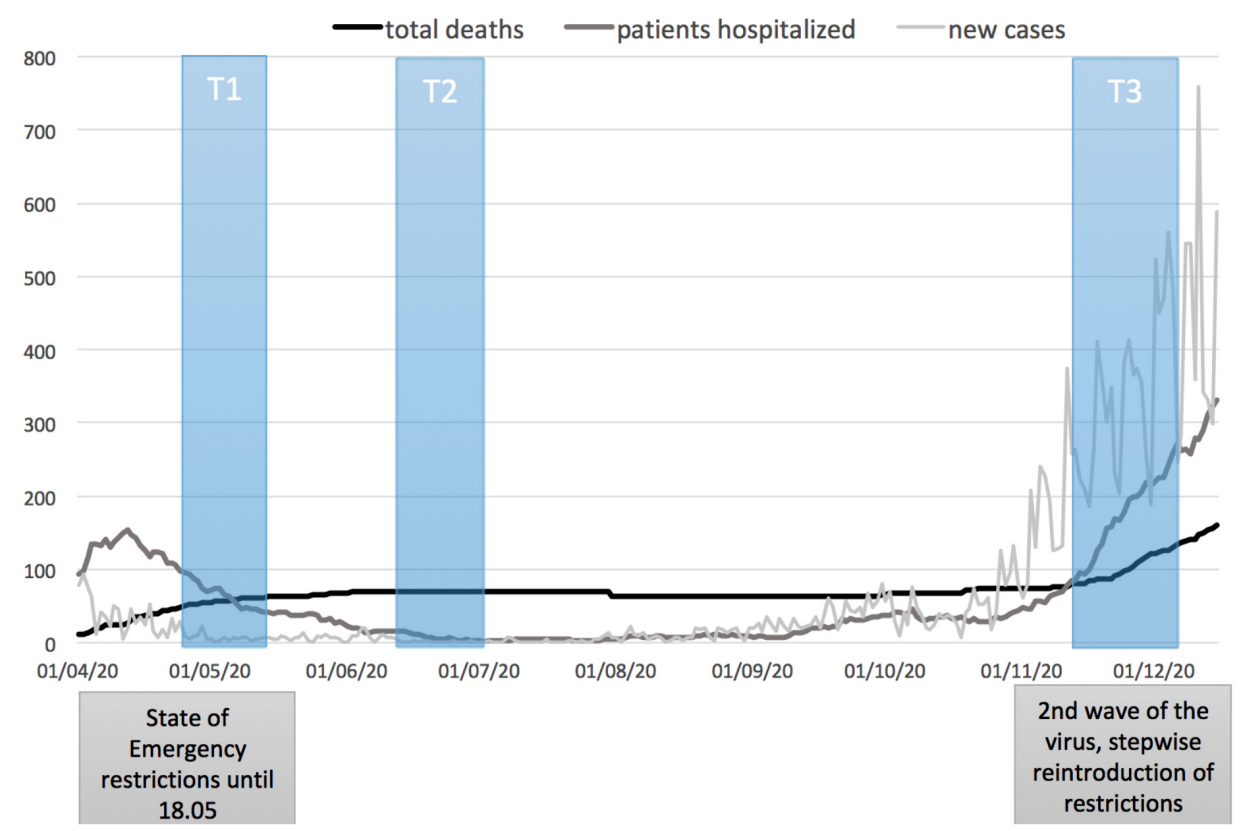

Figure 1. Situation during three recruitment waves (T1-T3): the number of COVID-19 deaths, patients hospitalized, new cases per day and periods of restrictions.

COVID-19 infection was diagnosed in almost $0.2 \%$ of the population in the first half of 2020. As with any other infectious disease, notification of only laboratoryconfirmed cases underestimates the actual incidence in the population (as clinically diagnosed cases and asymptomatic cases are not included). The number of COVID-19 cases is also related to the testing guidance. Until April 8th, primarily the elderly and people with comorbidities were tested. From April 8th, testing was extended to all people with acute upper respiratory infections.

The survey included information about the study, a set of demographic, health and wellness questions, and a set of self-reported scales about mental health and wellbeing. Demographics were only collected at the first wave. Some questions were modified or added in T2 and T3 data collections depending on the COVID-19 situation in the country. Participants' answers were anonymous and it took approximately 30 minutes to complete the questionnaire. The study was conducted in concordance with the Declaration of Helsinki and approved by the Ethics Committee of Tallinn University, Estonia.

\subsection{Instruments}

Emotional State Questionnaire-2. EST-Q2 (Ööpik et al. 2006) is a self-report questionnaire to assess symptoms of depression, anxiety, and symptoms related to depression and anxiety. The questionnaire consists of 28 items rated on a 5-point Likert scale $(1=$ not at all to $5=$ all the time) in six subscales: Depression (cut off 
point $>11$ ), Anxiety ( $>11$ ), Agoraphobia-Panic ( $>6$ ), Social Anxiety ( $>3$ ), Fatigue $(>6)$ and Insomnia $(>5)$. EST-Q2 is reflecting symptoms of depressive and anxiety disorders according to ICD-10 and DSM-IV during the previous month. Internal consistency of the scales is $\alpha=.69-.88$.

Alcohol Use Disorders Identification Test. AUDIT-C (WHO 2001) is a 3-item alcohol screening instrument that can help identify patients who are hazardous drinkers or have active alcohol use disorders (including alcohol abuse or dependence). The AUDIT-C is scored on a scale of $0-12$; a score of 5 or more in men (or 4 in women) is considered positive, optimal for identifying hazardous drinking or active alcohol use disorders.

Perceived Stress Scale (PSS; Cohen et al. 1983) is a self-report measure designed to assess the degree to which individuals appraise experiences as difficult to manage and overwhelming. Participants are asked to indicate on a five-point scale $(0=$ never, $4=$ very often) how often they felt or thought a certain way during the last 4 weeks (e.g., "How often did you feel unable to control the important things in life?"). We used the shortened version of this scale consisting of 10 items rated on a 5-point Likert scale $(0=$ never to $4=$ very often; items $4,5,7$ and 8 will be reversed $)$. The PSS total score was obtained by summing the scale items. This scale has demonstrated good internal reliability $(\alpha=.84-.86$, Cohen et al. 1983).

The PTSD Checklist Civilian version (PCL-C; Weathers et al. 1993) is a self-report rating scale for assessing post-traumatic stress disorder (PTSD). The PCL-C consists of 17 items which correspond to the DSM-IV symptoms of PTSD. Respondents are instructed to indicate how much they have been bothered by each symptom in the past month using a 5 -point Likert scale $(1=$ not at all to $5=$ extremely). Internal consistency is good, with Cronbach's $\alpha=.97$ (Weathers et al. 1993).

\subsection{Statistical analyses}

The procedure of DeVellis (2003) was used to translate PSS and PCL-C into the Estonian language. The items were translated into Estonian by two persons. An expert group consisting of three persons compared the translations and a final set of items was selected. The items were translated back into English by one person and compared to the original items. If necessary, then corrections were made.

To examine differences between waves across instruments, repeated measures ANOVA-s with a Greenhouse-Geisser correction were used (in pairwise comparisons Bonferroni correction was used). To examine the associations of constructs with age (at T1), Pearson's correlations and partial correlations (with partialization of the score from previous data collection wave) were used. Normal distribution was not observed for all the variables, but these methods were still used because they are rather robust to violations of normality (Norman 2010). The scores of EST-Q2 subscales and AUDIT-C scales were also dichotomized (scores below or above cut-off points) based on the cutoff scores. The differences between waves across the dichotomized scores were analyzed using McNemar tests. SPSS 27.0 was used to conduct the statistical analyses. Results yielding a $p<0.05$ were considered statistically significant. 


\section{Results}

\subsection{Exposure with COVID-19}

At T1 6\% of the respondents have been tested for COVID-19 at least once. By T2 the proportion had doubled being $13.5 \%$ and by T3 it was already nearly half, $45 \%$. At T1 and T2 no respondents but by T3 one respondent was diagnosed COVID-19 positive. At T1 and T2 only some respondents ( $n=3$ and $n=2$, respectively) answered that someone they know had been diagnosed positive in COVID-19 but at T3 already $38 \%$ respondents indicated that their family member or someone they know had and $18 \%$ that they are currently COVID-19 positive.

\subsection{The differences between scales in three data collection waves}

As the psychometric properties of the PSS and PCL-C scales in Estonian have not been measured, the psychometric data are presented using the results from T1. For the PSS, Kaiser-Meyer-Olkin's index KMO $=.871(p<.001)$ indicated that the sample was large and had enough power to conduct exploratory factor analysis. Next, principal component analysis with varimax rotation (communalities and factor loadings) and reliability (if item deleted) analysis was performed. The number of factors was set to 1 . According to principal component analysis, the one-component solution explained $50.60 \%$ of the variance of data (loadings between $.56-.80$, see descriptive statistics from Table 1).

For the PCL-C, Kaiser-Meyer-Olkin's index KMO =.923 $(p<.001)$ indicated that the sample was large and had enough power to conduct exploratory factor analysis. According to principal component analysis, the one-component solution explained $48.27 \%$ of the variance of data (loadings between .61 - .77).

Then repeated measures ANOVA-s were applied to examine differences between time-points across instruments (see Table 1). There were no differences between three time-points in Depression, Social Anxiety and Insomnia subscores, and in the score of AUDIT-C. Following significant differences emerged.

There was a significant difference in Anxiety score, $F(1.896,364.104)=4.70$, $p<.01, \eta^{2}=.024,1-\beta=.770$. Post-hoc test indicated that Anxiety scores were higher at T3 compared to T2 $(p<.01)$. There was a significant difference in Agoraphobia and panic score, $F(1.788,345.128)=5.30, p<.01, \eta^{2}=.027,1-\beta=.804$. Post-hoc test indicated that Agoraphobia and panic scores were higher at T1 compared to T3 $(p<.05)$. There was a significant difference in Fatigue score, $F(1.946,375.635)$ $=5.14, p<.01, \eta^{2}=.026,1-\beta=.816$. Post-hoc test indicated that Fatigue scores were higher at T3 compared to T2 $(p<.01)$. There was a significant difference in Perceived stress score, $F(1.938,389.625)=3.98, p<.05, \eta^{2}=.019,1-\beta=.702$. Post-hoc test indicated that Perceived stress scores were lower at T2 compared to T1 $(p<.05)$. Finally, there was a significant difference in PCL-C score, $F(1.998,365.721)$ $=4.71, p<.01, \eta^{2}=.025,1-\beta=.787$. Post-hoc test indicated that PCL-C scores were higher at T1 compared to T2 $(p<.05)$. 


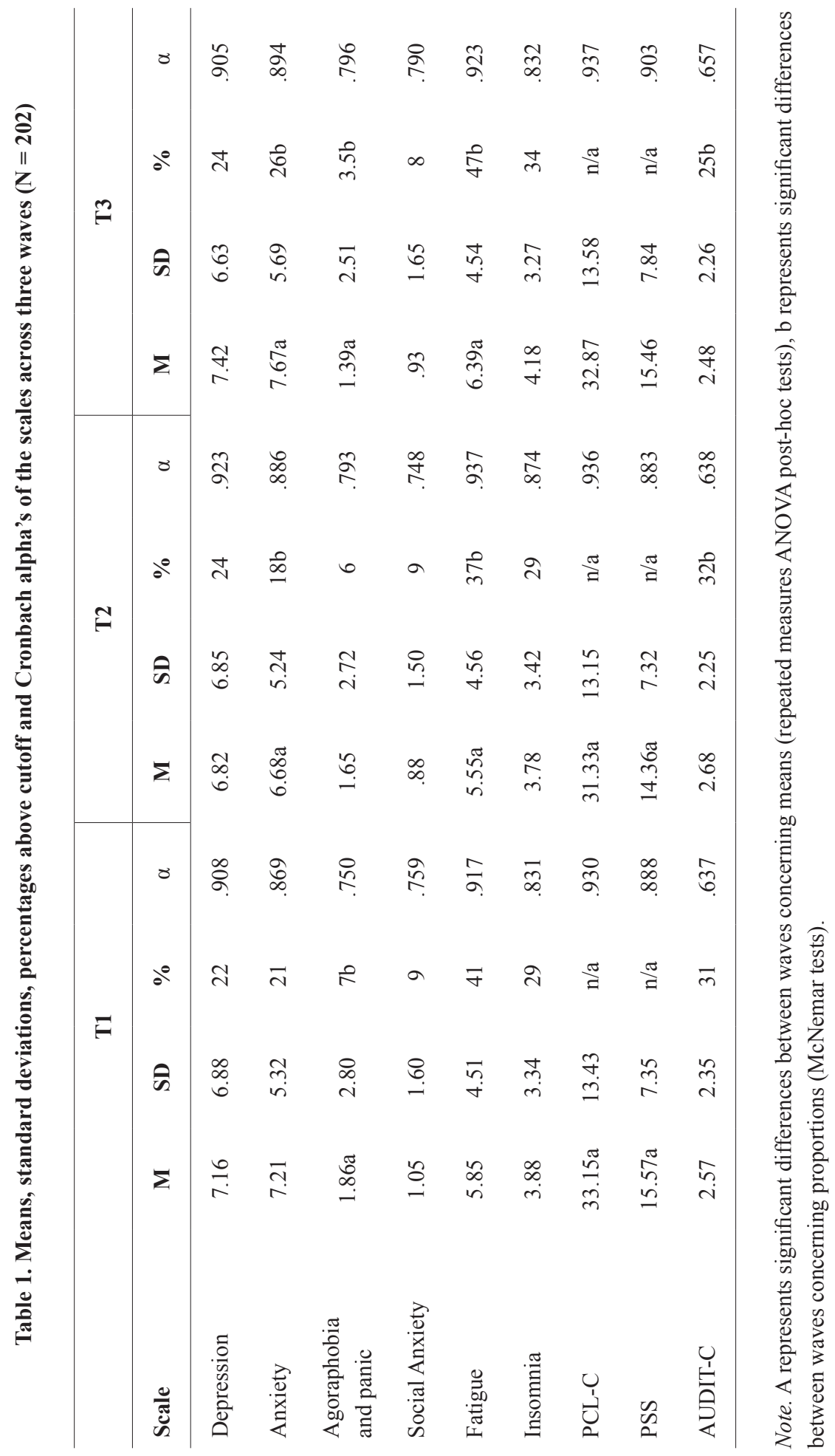


Table 2. Associations between mental health constructs and age across three time-points ( $\mathrm{N}=$ 202). Pearson's zero-order correlations and partial correlations (in brackets) with partialization of the previous time-point $(T)$ scores of the respective scale

\begin{tabular}{lccc}
\hline Scale & T1 & T2 & T3 \\
\hline Depression & $-.159^{*}$ & $-.173^{* *}(-.083)$ & $-.253^{* *}\left(-.188^{* *}\right)$ \\
Anxiety & $-.230^{* *}$ & $-.185^{* *}(-.019)$ & $-.247^{* *}\left(-.166^{* *}\right)$ \\
Agoraphobia and panic & -.022 & $-.030(.057)$ & $-.018(-.006)$ \\
Social Anxiety & $-.152^{*}$ & $-.086(-.023)$ & $-.106(-.074)$ \\
Fatigue & $-.233^{* *}$ & $-.176^{* *}(-.005)$ & $-.175^{* *}(-.078)$ \\
Insomnia & .004 & $-.011(-.020)$ & $.008(.025)$ \\
PCL-C & .015 & $.024(.020)$ & $-.010(-.045)$ \\
PSS & $-.166^{* *}$ & $-.172^{* *}(-.086)$ & $-.173^{* *}(-.091)$ \\
AUDIT-C & -.100 & $-.086(-.002)$ & $-.008(.112)$ \\
\hline
\end{tabular}

Note. ${ }^{*}$ Significant at .05 level; $* *$ significant at .01 level

Now the differences between waves for EST-Q2 and AUDIT-C scales were analysed using McNemar tests. Following significant differences were present. At T3 there were a larger proportion of respondents whose Anxiety and Fatigue scores were above the cut-off point compared to T2 ( $p$-s $<.01$, respectively). Finally, at $\mathrm{T} 2$ there were a larger proportion of respondents whose AUDIT-C score was above the cut-off point compared to T3 $(p<.01)$. At T1 there was a larger proportion of respondents whose Agoraphobia and panic score was above the cut-off point compared to T3 $(p<.05)$.

\subsection{The associations with age as risk factor}

The associations of participants' age (at T1) as a potential risk factor with absolute values of different scales at single time-points were assessed by using Pearson's correlations (Table 2). The older the respondents were, the lower were their Depression, Anxiety, Fatigue and Perceived stress scores at all three time-points. At the first time-point, the older the respondents, the lower the Social anxiety scores.

Finally, Pearson's partial correlations were used to examine the associations of age with the relative changes on different scales over time (Table 2). Thus, in all partial correlations the previous values (T1-values for T2, and T2-values for T3) of the 
respective scale, were partialized. There was significant negative partial correlation between age and Depression score at T3 whilst controlling for Depression score at T2. Similarly, there was a significant negative partial correlation between age and Anxiety score at T3 whilst controlling for Anxiety score at T2. This indicates that in the autumn, older age was also associated with lower Depression and Anxiety scores relative to baseline scores in the summer.

\section{Discussion}

This is the first Estonian longitudinal study that maps changes in mental health during the COVID-19 pandemic. T1 data collection was conducted when the SoE in Estonia was in force during the first wave of COVID-19 pandemic, T2 after the SoE ended and T3 when the second wave of COVID-19 pandemic was in increase. The article helps to broaden our understanding of trends and contributing factors of COVID-19 in 2020 in Estonia. Additionally, this study provides new insight into the longitudinal fluctuations of mental health during the second wave of pandemic in late autumn 2020.

At T1 people were faced with a completely new situation of their lifetime - this was demonstrated by the highest prevalence of EST-Q2 panic scores (compared to T3) and increased scores in PSS and PCL-C (compared to T2). The novelty of the situation and lack of adequate knowledge can provide an explanation for why during the $\mathrm{T} 1$ the level of perceived stress and symptoms of post-traumatic stress disorder were the highest of three time-points. People were afraid of what was to come (indicated by the increased panic and agoraphobia score) and struggled with pandemic-related restrictions. Public health actions, such as social distancing are necessary to reduce the spread of COVID-19, but they can increase the psychological stress that may not only have undesirable impact on individuals, but also cause social and economic problems.

By T2, the first wave of COVID-19 was over in Estonia and thus the level of different parameters we measured were the lowest. However, the improvement was statistically non-significant for depression, anxiety and other related (EST-Q2) symptoms. Although a statistically significant improvement in perceived and posttraumatic stress after the first wave of COVID-19 was observed, the effect sizes were very small, indicating low practically significant improvement.

By T3 (along with the presence of the second wave of COVID-19 pandemic), the anxiety and fatigue scores have increased along with a statistically non-significant reincrease of PSS and PCL-C scores compared to T2, which may indicate that by fall 2020 people may have become more tired of the pandemic as well as being more anxious (as the probability of getting infected increased). The impact of economic adversities (e.g., job loss or fear of losing the job) caused by the COVID-19 pandemic may also explain the increase of anxiety and fatigue.

At T2 there were a larger proportion of respondents whose AUDIT-C score was above the cut-off point compared to T3 (when the second wave of COVID-19 
pandemic was in increase). This finding goes contrary to previous studies indicating an increase in alcohol use due to higher levels of anxiety and depression symptoms (Stanton et al. 2020, Oksanen et al. 2020, Newby et al. 2020, Ahmed et al. 2020). However, it is reported to be a lowered level of consumption during the COVID-19 pandemic (Kilian et al. 2021). One interpretation of this finding could be that by autumn 2020, both reduced physical and financial availability of the alcohol may have affected alcohol consumption. Also, as the data on alcohol consumption is selfreported, it may therefore be an under- or over-estimate.

Since we recruited our sample after the start of pandemic, we could not directly compare our data to pre-COVID-19 levels and draw causal conclusions about the effect of pandemic on mental health indicators. Nonetheless, remarkably high proportions of respondents above cut-offs in our sample (e.g. compared to $6.6 \%$ for depressive symptoms in 2014 in the Estonian population - Arias-de la Torre et al. in press) allows us to plausibly suggest that COVID-19 pandemic did increase mental health risks in Estonia. Therefore, the general finding of this study is that COVID-19 pandemic in Estonia with its second wave has a continuous detrimental effect on mental health. The prevalence of depression, anxiety and their related symptoms did not decrease significantly during the 2020 (with exception of panic subscores) as compared to the initial stage of the pandemic, but rather increased further by late autumn together with the second wave of pandemic.

Previous longitudinal studies have observed reduction in distress close to prepandemic levels by June 2020 (Daly and Robinson 2021), and fairly rapid decline of depressive and anxiety symptoms after the lockdown (Fancourt et al. 2021). Also, meta-analysis across longitudinal studies has shown generally decreasing mental health symptoms by mid-2020 compared to the first two months of the pandemic (Robinson et al. 2021). Albeit the meta-analysis pointed out that depressive symptoms remained higher past the early phases of the pandemic, which can be supported by our Estonian results as well. Studies mentioned above suggested that possibly the initial consequences were followed by a period of psychological adaptation. Although we observed a slight improvement in perceived and posttraumatic stress after the first wave of pandemic, our data from three time-points across the year suggests relatively constant prevalence of mental health problems during 2020 in Estonia. Similar persistence was observed by Pieh and colleagues (2021) in Austrian population. They also found a slight improvement in perceived stress, but clinically relevant symptoms of depression, anxiety, or insomnia were not decreased by September 2020.

While stressors are affecting the whole population, the data suggests that older adults are managing the stress of the coronavirus pandemic better than younger adults, reporting less depression, anxiety, fatigue and perceived stress scores at all three time-points. Older age was also associated with lower symptoms of depression and anxiety in the autumn relative to baseline scores in the summer. This indicates that younger adults may have been more vulnerable for prolonged symptoms of depression and anxiety during the autumn 2020 when the second wave of pandemic started in Estonia. These results could be explained by the fact that young people 
have less financial security and social stability. The finding is in compliance with the general finding (non-pandemic) that younger people are more vulnerable to mental health problems (Twenge et al. 2019). While we cannot rule out alternative explanations, the results of this study suggest that age may offer a buffer against negative feelings brought on by the virus's impact. Our findings resonate with results from surveys conducted in other countries (Wilson et al. 2020, Savolainen et al. 2021, Pierce et al. 2020, Kwong et al. 2020, Gonzalez-Sanguino et al. 2021).

As for limitations, we have to bear in mind that our sample was not representative of Estonia's population, so we have to be careful when generalizing our conclusions. Most concerning is the under-representation of males (9.4\%) in our sample. Special cautiousness is needed when making inferences for males as most of the respondents were married women in higher education. Thus, the results of this study should be seen in response to who were the respondents. Another limitation in our study is the attrition of participants throughout the assessments. Survivorship bias in a longitudinal mental health survey during pandemic has shown to produce overly optimistic interpretations of mental health trends over time (Czeisler et al. 2021), which may have possibly biased our results as well.

In conclusion, the prevalence and changes in different domains of mental health such as the scores of depression, anxiety, perceived and post-traumatic stress, and the use of alcohol concerning COVID-19 has varied considerably between different countries. Estonia is no exception to this situation. Therefore, as these environmental characteristics seem to differ considerably between the countries, it is important to report findings among different populations. As the COVID-19 pandemic continues, the full extension of the impact of COVID-19 on mental health is yet to be established. However, the collected data from different countries provide an opportunity to link the impact of pandemic to changes in mental health, and to inform the management of future crises.

\section{Acknowledgements}

This research has been supported by the Project TA2620 Tallinn University Research Fund ("The Centre of Excellence in Behavioural and Neural Science") and the Tallinn University ASTRA project ("TU TEE - Tallinn University as a promoter of intelligent lifestyle"). We wish to thank our research group members Aleksander Pulver, Kadi Liik, Avo-Rein Tereping and Valeri Murnikov for helping to prepare this study. 
Addresses:

Karel Kulbin

Kristjan Kask (corresponding author)

Kristiina Uriko

Centre of Excellence in Behavioural and Neural Sciences

School of Natural Sciences and Health

Tallinn University

Narva mnt 25

Tallinn, 10120, Estonia

E-mail:kask@tlu.ee

Tel.: +3726409473

\section{References}

Ahmed, M. Z., O. Ahmed, Z. Aibao, S. Hanbin, L. Siyu, and A. Ahmad (2020) "Epidemic of COVID-19 in China and associated psychological problems". Asian Psychiatry Journal 51, 102092. Doi: 10.1016/j.ajp.2020.102092

Arias-de la Torre, J., G. Vilagut, A. Ronaldson, A. Serrano-Blanco, V. Martín, M. Peters, ... and J. Alonso (in press) "Prevalence and variability of current depressive disorder in 27 European countries: a population-based study". The Lancet Public Health. Doi: https://doi.org/10.1016/ S2468-2667(21)00047-5

Bendau, A., J. Plag, S. Kunas, S. Wyka, A. Ströhle, and M. B. Petzold (2021) "Longitudinal changes in anxiety and psychological distress, and associated risk and protective factors during the first three months of the COVID-19 pandemic in Germany". Brain and Behavior 11, 2, e01964. Doi: https://doi.org/10.1002/brb3.1964

Cohen, S., T. Kamarck, and R. Mermelstein (1983) "A global measure of perceived stress". Journal of Health and Social Behavior 24, 386-396.

Daly, M. and E. Robinson (2021) "Psychological distress and adaptation to the COVID-19 crisis in the United States". Journal of Psychiatric Research 136, 603-609. Doi: 10.1016/j. jpsychires.2020.10.035

Daly, M., A. R. Sutin, and E. Robinson (2020) "Longitudinal changes in mental health and the COVID-19 pandemic: evidence from the UK Household Longitudinal Study". Psychological Medicine 13, 1-10. Doi: https://doi.org/ 10.1017/S0033291720004432

DeVellis, R. F. (2003) Scale development: theory and applications. 2nd ed. California: SAGE Publications Inc.

Fancourt, D., A. Steptoe, and F. Bu (2021) “Trajectories of anxiety and depressive symptoms during enforced isolation due to COVID-19 in England: a longitudinal observational study". The Lancet Psychiatry 8, 141-149. Doi: 10.1016/S2215-0366(20)30482-X

González-Sanguino, C., B. Ausín, M. A. Castellanos, J. Saiz, and M. Muñoz (2021) "Mental health consequences of the Covid-19 outbreak in Spain: a longitudinal study of the alarm situation and return to the new normality". Progress in Neuro-Psychopharmacology and Biological Psychiatry 107, 110219. Doi: https://doi.org/10.1016/j.pnpbp.2020.110219 
Hale, T., S. Webster, A. Petherick, T. Phillips, and B. Kira (2020) Oxford COVID-19 government response tracker. Blavatnik School of Government, University of Oxford. Available online at $<$ https://www.bsg.ox.ac.uk/research/research-projects/oxford-covid-19-government-responsetracker>. Accessed on 05.08. 2021.

Holmes, E. A., R. C. O’Connor, V. H. Perry, I. Tracey, S. Wessely, L. Arseneault, C. Ballard, H. Christensen, R. Cohen Silver, I. Everall, T. Ford, A. John, T. Kabir, K. King, I. Madan, S. Michie, A. K. Przybylski, R. Shafran, A. Sweeney, ... and E. Bullmore (2020) "Multidisciplinary research priorities for the COVID-19 pandemic: a call for action for mental health science". The Lancet Psychiatry 7, P547-560. Doi: https://doi.org/10.1016/S22150366(20)30168-1

Keyes, K. M., M. L. Hatzenbuehler, and D. S. Hasin (2011) "Stressful life experiences, alcohol consumption, and alcohol use disorders: the epidemiologic evidence for four main types of stressors". Psychopharmacology 218, 1-17. Doi: 10.1007/s00213-011-2236-1

Kilian, C., J. Rehm, P. Allebeck, F. Braddick, A. Gual, M. Barták, K. Bloomfield, A. Gil, M. Neufeld, A. O'Donnell, B. Petruželka, V. Rogalewicz, B. Schulte, J. Manthey, with the European Study Group on Alcohol Use and COVID-19 (2021) "Alcohol consumption during the COVID-19 pandemic in Europe: a large-scale cross-sectional study in 21 countries". Addiction. Doi: https://doi.org/10.1111/add.15530

Kousoulis, A., T. Van Bortel, P. Hernandez, and A. John (2020) "The long term mental health impact of COVID-19 must not be ignored”. BMJ Opinion. Available online at $<$ https://blogs.bmj. $\mathrm{com} / \mathrm{bmj} / 2020 / 05 / 05 /$ the-long-term-mental-health-impact-of-covid-19-must-not-be-ignored/>. Accesed on 03.05.2021.

Kwong, A. S., R. M. Pearson, M. J. Adams, K. Northstone, K. Tilling, D. Smith, ... and N. J. Timpson (2020) "Mental health during the COVID-19 pandemic in two longitudinal UK population cohorts." medRxiv. Doi: https://doi.org/10.1101/2020.06.16.20133116

Newby, J. M., K. O’Moore, S. Tang, H. Christensen, and K. Faasse (2020) "Acute mental health responses during the COVID-19 pandemic in Australia”. PloS One 15, e0236562. Doi: https:// doi.org/10.1371/journal.pone.0236562

Norman, G. (2010) "Likert scales, levels of measurement and the 'laws' of statistics". Advances in Health Sciences Education 15, 625-632.

Oksanen, A., I. Savolainen, N. Savela, and R. Oksa (2020) "Psychological stressors predicting increased drinking during the COVID-19 crisis: a longitudinal national survey study of workers in Finland". Alcohol and Alcoholism 56, 299-306. Doi: 10.1093/alcalc/agaa124

Ööpik, P., A. Aluoja, R. Kalda, and H. Maaroos (2006) "Screening for depression in primary care". Family Practice 23, 693-698.

Parm, Ü., A. Aluoja, T. Tomingas, and A.-L. Tamm (2021) "Impact of the COVID-19 pandemic on Estonian elite athletes: survey on mental health characteristics, training conditions, competition possibilities, and perception of supportiveness". International Journal of Environmental Research and Public Health 18, 4317. Doi: 10.3390/ijerph18084317

Pieh, C., S. Budimir, E. Humer, and T. Probst (2021) "Comparing mental health during the COVID-19 lockdown and 6 months after the lockdown in Austria: a longitudinal study". Frontiers in Psychiatry 12. Doi: 10.3389/fpsyt.2021.625973 
Pierce, M., H. Hope, T. Ford, S. Hatch, M. Hotopf, A. John, ... and K. M. Abel (2020) "Mental health before and during the COVID-19 pandemic: a longitudinal probability sample survey of the UK population". The Lancet Psychiatry 7, 10, 883-892. Doi: https://doi.org/10.1016/S22150366(20)30308-4

Robinson, E., A. R. Sutin, M. Daly, and A. Jones (2021) “A systematic review and meta-analysis of longitudinal cohort studies comparing mental health before versus during the COVID-19 pandemic". medRxiv. Doi: https://doi.org/10.1101/2021.03.04.21252921

Savolainen, I., R. Oksa, N. Savela, M. Celuch, and A. Oksanen (2021) "COVID-19 anxiety - a longitudinal survey study of psychological and situational risks among Finnish workers". International Journal of Environmental Research and Public Health 18, 794. DOI: https://doi. org/10.3390/ijerph18020794

Stanton, R., Q. G. To, S. Khalesi, S. L. Williams, S. J. Alley, T. L. Thwaite, A. S. Fenning, and C. Vandelanotte (2020) "Depression, anxiety and stress during COVID-19: associations with changes in physical activity, sleep, tobacco and alcohol use in Australian adults". International Journal of Environmental Research and Public Health 17, 4065. Doi: https://doi.org/10.3390/ ijerph17114065

Twenge, J. M., A. B. Cooper, T. E. Joiner, M. E. Duffy, and S. G. Binau (2019) “Age, period, and cohort trends in mood disorder indicators and suicide-related outcomes in a nationally representative dataset, 2005-2017'. Journal of Abnormal Psychology 128, 185-199. https://doi.org/10.1037/ abn0000410

Weathers, F. W., B. T. Litz, D. S. Herman, J. A. Huska, and T. M. Keane (1993) "The PTSD checklist (PCL): reliability, validity, and diagnostic utility". In Proceedings of the Annual Conference of the International Society for Traumatic Stress Studies. San Antonio, Texas.

Wilson, J. M., J. Lee, and N. J. Shook (2020) "COVID-19 worries and mental health: the moderating effect of age". Aging and Mental Health. Doi: doi.org/10.1080/13607863.2020.1856778

World Health Organization (2020) Coronavirus disease 2019 (COVID-19). (Situation Report, 91.) World Health Organization, Geneva. Available online at $<$ https://www.who.int/emergencies/ diseases/novel-coronavirus-2019/situation-reports>. Accessed on 05.08.2021.

World Health Organization (2001) AUDIT: the Alcohol Use Disorders Identification Test: guidelines for use in primary health care. Thomas F. Babor et al. 2nd ed. World Health Organization. Available online at $<$ https://apps.who.int/iris/handle/10665/67205>. Accessed on 05.08.2021. 